PAPER

\title{
Pontine atrophy precedes cerebellar degeneration in spinocerebellar ataxia 7: MRI-based volumetric analysis
}

\author{
O Y Bang, P H Lee, S Y Kim, H J Kim, K Huh
}

J Neurol Neurosurg Psychiatry 2004;75:1452-1456. doi: 10.1136/jnnp.2003.029819

See end of article for authors' affiliations

.....................

Correspondence to:

K Huh, MD, Department of

Neurology, College of

Medicine, Ajou University,

Woncheon-dong San 5,

Paldal-ku, Suwon,

Kyungki-do, 442-749,

Korea;

nmboy@unitel.co.kr

Received 7 October 2003

In revised form

6 January 2004

Accepted 16 January 2004

\begin{abstract}
Background and objective: Spinocerebellar ataxia 7 (SCA7) is characterised by cerebellar ataxia and visual loss. The aim of the present study was to elucidate the magnetic resonance imaging (MRI) findings characteristic of patients with SCA7.

Methods: Twenty patients with SCA (eight SCA3, three SCA6, and nine SCA7) and 20 control subjects underwent an MRI-based volumetric analysis.

Results: The pontine volume in patients with SCA7 was decreased by a greater amount than in patients with other types of SCA $(p<0.01)$, whereas the cerebellar volume was not different from that in other types of SCA ( $p>0.05)$. Pontine atrophy was a consistent finding in all patients with SCA7 regardless of the degree of cerebellar atrophy or the severity or duration of illness. In contrast, cerebellar atrophy was not found in those with a short duration of illness or mild ataxia, but became prominent as the severity and duration of illness progressed.

Conclusions: Our study suggests that neurodegeneration is ongoing during the life of individuals with SCA7, and that the primary pathology in these individuals involves the brainstem rather than the cerebellum. In addition, pontine atrophy is a prominent, consistent finding in SCA7, and may help in establishing the clinical diagnosis of SCA7.
\end{abstract}

$\mathrm{S}$ pinocerebellar ataxia type 7 (SCA7) is a neurodegenerative disorder caused by a CAG/polyglutamine repeat expansion mutation in the gene encoding the ataxin 7 protein. This type of mutation is known to cause nine inherited neurodegenerative disorders, Huntington's disease (HD), dentatorubral pallidoluysian atrophy (DRPLA), and SCA 1, 2, 3, 4, 5, 6, and 7.

SCAs form a heterogeneous group of dominantly inherited disorders characterised by progressive ataxia that results from degeneration of the cerebellum and its afferent and efferent connections. Although cerebellar atrophy is a consistent feature in most symptomatic SCA patients, the involvement of extracerebellar structures varies with the type of SCA. Multiregional brain hypometabolism has been observed in SCA, even in SCA6, ${ }^{2}$ in which selective cerebellar atrophy is consistently observed, ${ }^{3}$ suggesting that the brain dysfunction associated with SCAs is not limited to the cerebellum. However, the initial site of involvement in each type of SCA remains unclear.

With the availability of magnetic resonance imaging (MRI), it has become possible to study the brain morphology in SCA in vivo. Previous MRI studies revealed significant differences in the pattern and extent of the morphological alterations with different SCA mutations. There is more severe cerebellar and brainstem atrophy in SCA2 than in SCAl and SCA $3,{ }^{45}$ and there is relatively selective involvement of the cerebellum in SCA6. ${ }^{3}$ MRI-based volumetric analysis appears to be of particular importance because the neuropathological abnormalities observed in postmortem examinations may only reflect far advanced pathological changes. To the best of our knowledge, MRI-based volumetric analysis of patients with SCA7 has not been carried out previously.

This study elucidates the characteristic MRI findings of SCA7 compared with other SCA types. We hypothesised that a morphometric analysis of patients with SCA7 could reveal the disease course in the early stage of cerebellar ataxia. Therefore, we conducted a volumetric analysis of nine genetically confirmed SCA7 patients, and compared the findings with those of other SCA patients and normal control subjects.

\section{METHODS}

We studied 20 patients manifesting progressive ataxia as the main clinical finding: nine patients with SCA7 from six families and 11 patients with other types of SCA (eight SCA3 and three SCA6). Informed consent was obtained from all enrolled patients.

For the molecular genetic studies, previously published primer sequences and conditions for the polymerase chain reaction were used to quantify the trinucleotide repeats of SCA 1, 2, 3, 6, 7, and DRPLA. ${ }^{6-11}$ We also screened the patients who lacked an expanded pathological allele and had a maternal inheritance pattern for known mutations associated with mitochondrial encephalopathy with ragged red fibre (MERRF), as described previously. ${ }^{12}{ }^{13}$ We excluded patients with a clinical diagnosis of possible or probable multisystem atrophy following the consensus statement published by Gilman et $a l^{14}$ and those with MERRF gene mutations. Twenty age and sex matched control subjects with no evidence of organic brain disease, such as migraine or tension headache, were selected for determination of reference values for morphometric analysis.

The neurological examination carried out had a specific focus on certain clinical features of brainstem involvement (pyramidal and extrapyramidal symptoms, voluntary and involuntary slow saccades, and dysphagia), and severity of ataxia. The results were graded as follows: I, walks without help; II, walks with some help; III, needs help to walk; IV, needs help to stand; V, bed-ridden.

The subjects were examined using 1.5-T MRI. MRI based volumetric analysis was performed according to previously

Abbreviations: DRPLA, dentatorubral pallidoluysian atrophy; $H D$, Huntington's disease; MRI, magnetic resonance imaging; SCA, spinocerebellar ataxia 
published methods. ${ }^{5}{ }^{15}$ The axial and sagittal Tl-weighted magnetic data (repetition time, $\mathrm{TR}=500 \mathrm{~ms}$; echo time, $\mathrm{TE}=8 \mathrm{~ms}$; slice thickness, $4 \mathrm{~mm}$; gap, $1 \mathrm{~mm}$ ) were processed using a commercially available computer workstation (Scion Image Beta 4.02; Scion Corp, Frederick, MD). Brain segmentation was performed manually on all controls and patients with SCA by a neuroradiologist who was blinded to the clinical data. The pons was segmented first. The pontine volume was calculated by summation and linear interpolation of the segmented axial slices. All slices between the inferior limit of segmentation, set at the lowest point of the pons, and the superior point of the pons were measured. The pons and cerebellum were separated by transecting the cerebellar peduncles along the plane of their entrance into the pons, namely at the shortest segment between the anterior recess of the fourth ventricle and the junction between the lateral border of the peduncles and the cerebellar cortex. Using this technique, the peduncles were largely excluded from the pontine volume. The cerebellar volume was calculated by summation and linear interpolation of the segmented sagittal slices. The pontine and cerebellar volumes were computed by multiplying the measured area per slice by the section thickness. Both volumes were normalised to the total intracranial volume, measured in cross-section, to reduce interindividual variation ${ }^{15}$ and are expressed as the percentage of the respective total intracranial volume. Other areas of the brain were not measured.

The differences between the groups were analysed using the Kruskal-Wallis H-test with the Mann-Whitney U-test as a post hoc analysis. The relationships between the various clinical parameters and the pontine or cerebellar volume and between the CAG repeat length and the pontine or cerebellar volume were evaluated using a linear regression analysis for the SCA7 patients. Probability values less than 0.05 were accepted as significant.

\section{RESULTS}

Volumetric analysis was performed in nine SCA7 patients, eight SCA3 patients, three SCA6 patients, and 20 control subjects. There were no significant differences in the clinical features (age, duration of illness, age at onset, and severity of ataxia) of patients with SCA7 and those with other types of SCA $(\mathrm{p}>0.05)$.

The clinical and volumetric data for the nine patients with SCA7 are shown in table 1 . All the patients showed the typical pattern of autosomal dominant cerebellar ataxia and exhibited visual loss with pigmentary retinopathy on ophthalmoscopy. Extracerebellar symptoms, such as pyramidal and extrapyramidal symptoms, voluntary and involuntary slow saccades, and dysphagia, were found more frequently in patients with severe pontine atrophy $(r=0.722, \mathrm{p}<0.05)$. Of the extracerebellar symptoms, slowing of saccades was most frequently observed (table 1). However, an increased number of CAG repeats did not significantly influence the volume of the cerebellum $(r=0.256, \mathrm{p}>0.05)$ or pons $(r=0.024$, $\mathrm{p}>0.05)$.

Figure lA shows the similarity in the degree of pontine atrophy in patients with different severity of illness. The degree of pontine atrophy in a patient (case l, fig lA, left) with disease of 22 years duration and severe cerebellar symptoms (wheelchair bound) was similar to that of a patient (case 4, fig 1A, right) with disease of six years duration and mild cerebellar symptoms (walks without help) (pontine volume/total intracranial volume, $0.38 \% v 0.33 \%$ ).

Figure $1(B, C)$ show the correlation between pontine or cerebellar atrophy and the duration of illness or severity of ataxia in the patients with SCA7. The degree of cerebellar atrophy was significantly correlated with both the duration of illness $(r=0.664, \mathrm{p}<0.05$, fig $1 \mathrm{~B})$ and the severity of ataxia $(r=0.673, \mathrm{p}<0.05$, fig $\mathrm{lC})$. However, there was no significant correlation between pontine atrophy and any clinical parameters measured - that is, duration of illness, age of onset, or severity of ataxia ( $p>0.05$ in all cases). Pontine atrophy was consistently observed in all the patients with SCA7, including those with minimal ataxia and illness of very short duration. Conversely, cerebellar atrophy was not found in either of the two patients with illness of short duration $(\leqslant 4$ years) and in two of the three patients with mild ataxia (walks without help).

Figure 2 summarises the MRI findings of the 20 patients with SCA and the 20 control subjects. The volumes of both the cerebellum and pons differed between the patients with SCA7 and control subjects $(\mathrm{p}<0.001$ in both cases; $95 \%$ confidence interval (CI) of the difference, 1.822 to 3.54 and 0.348 to 0.493 , respectively) (fig 2A). Although the degree of pontine atrophy correlated with the cerebellar atrophy $(r=0.884, \mathrm{p}=0.004)$, pontine atrophy was the most consistent MRI finding in SCA7, regardless of the presence or absence of cerebellar atrophy (fig 2A).

The cerebellar volume did not differ between the patients with SCA7 and those with other types of SCA $(p=0.239)$ (fig 2B). In contrast, compared with the patients with other SCA types, the patients with SCA7 had significant pontine atrophy (SCA3 $v$ SCA7, $\mathrm{p}=0.002 ;$ SCA6 $v$ SCA7, $\mathrm{p}=0.009$ ).

\section{DISCUSSION}

\section{Clinical implications of our findings}

SCA7 is characterised by cerebellar ataxia and visual loss, and some characteristic clinical features of molecularly confirmed SCA7 have been reported previously. ${ }^{16}$ However, the diagnosis of SCA7 based on clinical assessment has limited accuracy because there is marked variation in the age of onset, initial symptoms, and associated signs. ${ }^{16}$ It has been reported that decreased visual acuity and pigmentary macular degeneration are not necessarily found in patients with SCA $7^{16}$ and may be observed in other SCAs. ${ }^{17}$

In this respect, MRI may be helpful in revealing significant differences in the pattern and extent of the morphological alteration in SCA7 and other SCAs. In this study, we observed severe pontine atrophy in the patients with SCA7; this was much more prominent than in other types of SCA (SCA3 and SCA6) and was consistently found in patients with varying degrees of cerebellar atrophy and severity of illness. Pontine atrophy has been reported in other SCAs, even in SCA6, ${ }^{18}$ although it is quite unexpected for such patients to have pontine atrophy with their cerebellum spared, which we found in our patients with SCA7. All patients with SCA7 may not have the neuroradiological characteristics observed in this study, yet pontine atrophy was a prominent finding in our patients with SCA7. Our results suggest that assessment of pontine involvement on MRI substantiates the clinical diagnosis of SCA7. The difference between the ages of the patients with SCA7, (mean 40.7 (SD 12.8) years), those with other SCAs (37.9 (9.1) years), and normal control subjects (40.4 (11.2) years) did not affect the baseline volumetric data. The same was true for sex: SCA7 (male, 67\%), other SCAs (male, 45\%), and normal control subjects (male, 50\%).

In this study, both the pontine and cerebellar volumes were normalised to the total intracranial volume, rather than measuring each volume directly, because firstly, in degenerative diseases in which the whole brain is subject to atrophy, the total intracranial volume may provide the best available estimate of the premorbid brain volume, and secondly, measuring the total intracranial volume allows the whole brain and regional volumetric measures to be normalised for head size. The pontine volumes of the female control subjects included in this study were significantly lower than those of the male control subjects $(p=0.038)$, but 
Table 1 Clinical and neuroimaging findings of nine patients with spinocerebellar ataxia 7

\begin{tabular}{|c|c|c|c|c|c|c|c|c|}
\hline \multirow[b]{2}{*}{ Case } & \multirow{2}{*}{$\begin{array}{l}\text { No. of expanded } \\
\text { CAG repeats }\end{array}$} & \multirow[b]{2}{*}{ Sex/age } & \multirow{2}{*}{$\begin{array}{l}\text { Age at the } \\
\text { onset of } \\
\text { symptoms }\end{array}$} & \multirow{2}{*}{$\begin{array}{l}\text { Duration of disease } \\
\text { in years (age at walking } \\
\text { aid) }\end{array}$} & \multirow{2}{*}{$\begin{array}{l}\text { Ataxia } \\
\text { scale }\end{array}$} & \multirow{2}{*}{$\begin{array}{l}\text { Extracerebellar } \\
\text { symptoms }\end{array}$} & \multicolumn{2}{|c|}{$\begin{array}{l}\text { Volume of the posterior fossa } \\
\text { structures/total intracranial } \\
\text { volume }\end{array}$} \\
\hline & & & & & & & Pons (\%) & Cerebellum (\%) \\
\hline 1 & $45 / 10$ & $F / 59$ & 37 & $22(40)$ & 5 & Bulbar, EOM, PS & 0.38 & 7.14 \\
\hline 2 & $47 / 10$ & $M / 37$ & 32 & 4 (self ambulatory) & 1 & EOM & 0.63 & 10.47 \\
\hline 3 & $43 / 10$ & $\mathrm{~F} / 52$ & 39 & 13 (self ambulatory) & 1 & EOM, PS, EPS & 0.35 & 8.30 \\
\hline 4 & $47 / 10$ & $M / 40$ & 34 & $6(37)$ & 2 & Bulbar, EOM, PS, EPS & 0.33 & 8.19 \\
\hline 5 & $50 / 8$ & $M / 29$ & 22 & 7 (self ambulatory) & 1 & EPS & 0.38 & 7.80 \\
\hline 6 & $46 / 10$ & $M / 33$ & 29 & 4 (self ambulatory) & 1 & EOM, PS & 0.61 & 11.22 \\
\hline 7 & $44 / 12$ & $M / 59$ & 45 & $14(56)$ & 3 & Bulbar, EOM, PS, EPS & 0.34 & 8.66 \\
\hline 8 & $51 / 10$ & $F / 29$ & 15 & $13(22)$ & 4 & Bulbar, EOM, PS, EPS & 0.31 & 7.27 \\
\hline 9 & NC & $M / 28$ & 19 & $9(24)$ & 5 & EOM, PS & 0.44 & 8.08 \\
\hline
\end{tabular}

when we used this method of volumetric measurement, neither the pontine nor the cerebellar volumes differed as regards epidemiological factors such as age and sex.

Recently, Martin et al ${ }^{19}$ suggested that the term "mildly affected patient" should be reserved for those who show a definite degree of cerebellar atrophy without pontine involvement on MRI. However, they used visual inspection rather than volumetric analysis. In our volumetric analysis, pontine atrophy was a consistent finding, and we did not find a significant correlation between pontine atrophy and any of the clinical parameters measured or the CAG repeat length. Further studies with more subjects are needed to confirm possible relationships between the clinical parameters or CAG repeat length and the degree of pontine or cerebellar atrophy.
A

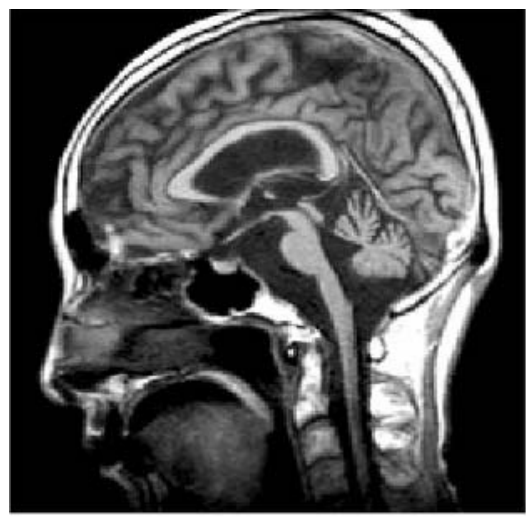

B
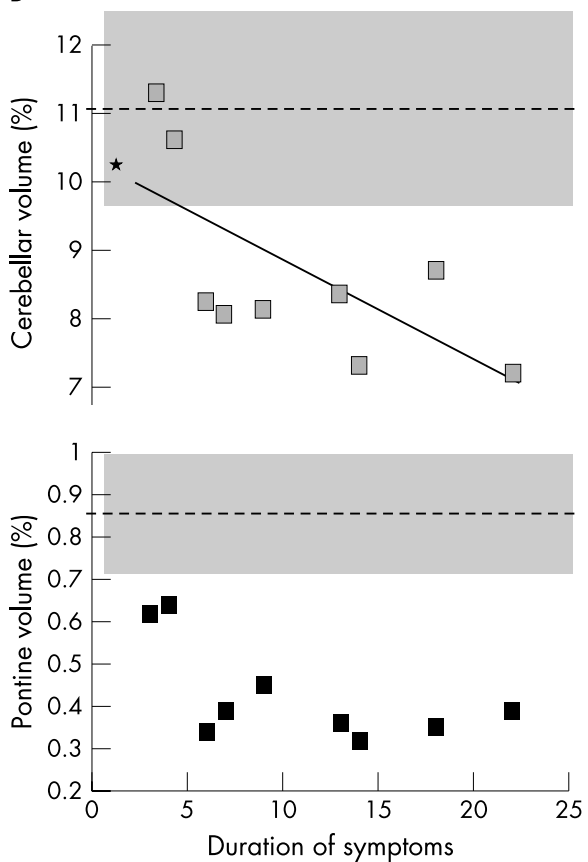

C
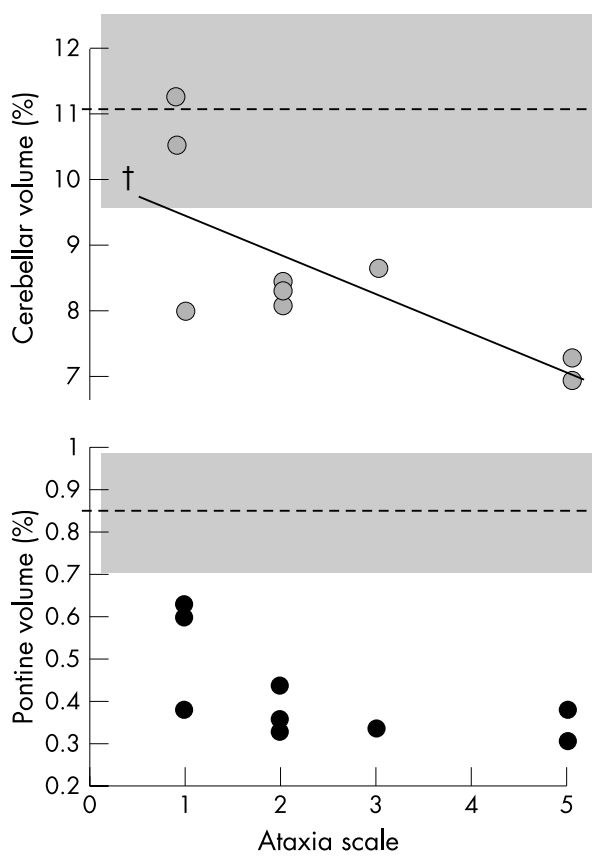

Figure 1 Correlation of clinical parameters with pontine and cerebellar volumes in nine patients with spinocerebellar ataxia 7. (A) Similar degree of pontine atrophy $10.38 \% \mathrm{v}$ $0.33 \%$ ) in patients with different severity of illness: (left) patient with long duration of disease and severe cerebellar symptoms and (right) patient with short duration of disease and mild cerebellar symptoms. Correlation of pontine and cerebellar volumes with (B) the duration and $(C)$ severity of illness. *Inverse correlation of cerebellar volume and the duration of illness. tlnverse correlation of cerebellar volume and the severity of illness. The grey area indicates the range of $2 \mathrm{SD}$ values of normal controls: cerebellum, mean 11.14 (range of 2 SD 9.5812.70); pons, mean 0.84 , (range of 2 SD 0.70-0.98). 


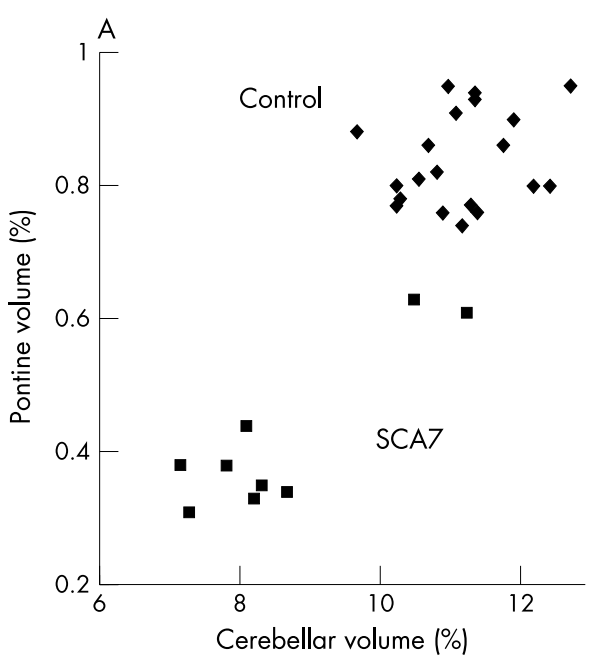

Neuropathological changes in patients with SCA7

The degenerative pattern may be specific enough to allow the diagnosis of SCA on neuropathological grounds. Our volumetric data are in good agreement with the neuropathological data of Martin et al, ${ }^{20}$ who found pontine atrophy to be a prominent feature in patients with SCA7. A neuropathological study of patients with SCA7, including a nearly asymptomatic patient, showed involvement of the pyramidal pathways and motor neurones of the brainstem and spinal cord, in addition to cerebellar atrophy affecting both the spinocerebellar and olivocerebellar tracts and the cerebellar cortex and efferent cerebellar pathways. ${ }^{20}$

Using an ataxin 7 immunoreactivity test, Lindenberg et al ${ }^{21}$ recently reported that the intensity of staining differed from region to region; staining was relatively strong in the inferior olive, dentate nucleus, and pontine nuclei, whereas the cerebellum, which was also affected, stained faintly. Moreover, Holmberg et al ${ }^{22}$ found very few nuclear inclusions in the cerebellum, a severely affected site in SCA7; ubiquitinated nuclear inclusions, a common characteristic in polyglutamine disorders, were frequently observed in the brainstem (14-24\%), but not in the cerebellum $(<1 \%)$. Our findings are in good agreement with this study in that pontine involvement rather than cerebellar atrophy was a consistent finding in SCA7. However, despite myriad studies of ataxin 7 distribution in the brain and non-central nervous system tissues of normal and SCA7 individuals, the distribution of ataxin 7 and the relation between ataxin 7 and nuclear inclusions remain controversial. ${ }^{23}$

\section{Distinct neuropathological pattern in SCA7}

SCA2 also shows pontine atrophy that is complete quite early during the course of SCA2; however, SCA2 differs from SCA7 in that it shows particularly widespread degeneration of neuronal populations, including regions thought to be typically affected in $\mathrm{HD}^{24}$

Unlike SCA7 and SCA2, SCA6 displays predominant Purkinje cell degeneration as the characteristic pathological feature, ${ }^{25}$ and shows neuronal degeneration confined to the cerebellar Purkinje cells and, to a lesser degree, the granular cells, with no structural involvement of other parts of the central nervous system. ${ }^{26}$ In the more common SCA3, the neuropathological findings of autopsied brains include neuronal cell loss in the dentate nucleus of the cerebellum, pontine nuclei, substantia nigra, and inner segment of the globus pallidus, and the preservation of the inferior olivary nucleus and Purkinje cells of the cerebellar cortex. ${ }^{27}$ In a case report, SCA3 showed a pattern consistently different from
SCA7, in that spinopontine involvement predominated over cerebellar involvement, no olivary involvement was seen, and the globus pallidus was clearly affected..$^{28}$

\section{Early involvement of the brainstem in patients with SCA7}

While SCA is diagnosed primarily from motor signs, especially ataxia, a recent study suggests that other signs, such as the slowing of saccades, ${ }^{29}$ precede motor disturbance as cognitive deficits in HD. ${ }^{30}$ The slowing of saccades has been reported to occur quite early in patients with $\mathrm{SCA}^{29}$ and SCA2, ${ }^{31}$ suggesting that the brainstem regions that mediate the generation of saccadic eye movements are a site of early neurodegeneration in both SCA7 and SCA2. Patients with SCA7 or SCA2 have been reported to show pontine atrophy. ${ }^{32}$ In our study, the slowing of voluntary and involuntary saccades was observed in eight of the nine patients with SCA7, independent of the duration or severity of illness.

Longitudinal studies using positron emission tomography (PET) or brain single photon emission computed tomography (SPECT) may help in understanding the timing and extent of the changes in the brain in patients with SCA7. Unfortunately, PET scanners are unavailable in most nuclear medicine laboratories, and brain SPECT imaging may underestimate pons abnormalities because of technical problems (the size of this structure and the resolution of the scintillation camera). We therefore conducted a volumetric analysis of patients with SCA7 of variable duration and severity of illness. Our results suggest that cerebellar atrophy is not found at the early symptomatic stage, but becomes prominent as the severity and duration of illness progress. A more important feature was that pontine atrophy was present in all patients with SCA7, including those in the early symptomatic stage. Our data for SCA7 contrast with the data on SCA3, in which atrophy develops in both the brainstem and cerebellum during the presymptomatic stage. ${ }^{33}$

\section{Limitations of the study}

This study has some limitations. First, we focused mainly on the changes in the pons and cerebellum and did not measure other intracranial structures. It should be mentioned that the atrophy of other structures of the central nervous system, such as the striatum, is reported to be a consistent neuropathological feature in the early stages of SCA2 and SCA3, but not in SCA7. Secondly, and most importantly, we did not perform an MRI morphometric analysis of asymptomatic gene carriers of SCA7. Until sufficient data from 
presymptomatic patients are accumulated, it will remain unresolved whether the disease progresses linearly from birth or develops as a multistage process with a long latent period followed by rapid progression of the dysfunctional stage. Lastly, we did not compare SCA7 with other types of SCA in which brainstem atrophy is reported to be a consistent feature, such as SCA2. ${ }^{4}$

\section{CONCLUSIONS}

In summary, our results suggest that neurodegeneration progresses continuously during the life of individuals with SCA7 and that the primary pathology may involve the brainstem, rather than the cerebellum. Although not all patients with SCA7 may have the neuroradiological characteristics observed in this study, pontine atrophy is a prominent finding in SCA7 and validates the clinical diagnosis of SCA7.

\section{ACKNOWLEDGEMENT}

We thank Professor Woon Ki Paik at Hanyang University for his advice and assistance in preparing the manuscript.

\section{Authors' affiliations}

O Y Bang, P H Lee, K Huh, Department of Neurology, School of Medicine, Ajou University, Korea

S Y Kim, Department of Neuroradiology, School of Medicine, Ajou University, Korea

H J Kim, Department of Medical Genetics, School of Medicine, Ajou University, Korea

Competing interests: none declared

\section{REFERENCES}

1 Margolis RL, Ross CA. Expansion explosion: new clues to the pathogenesis of repeat expansion neurodegenerative diseases. Trends Mol Med 2001;7:479-82.

2 Soong B, Liu R, Wu L, et al. Metabolic characterization of spinocerebellar ataxia type 6. Arch Neurol 2001;58:300-4.

3 Murata Y, Kawakami H, Yamaguchi S, et al. Characteristic magnetic resonance imagining findings in spinocerebellar ataxia 6. Arch Neurol 1998; $55: 1348-52$.

4 Burk K, Abele M, Fetter M, et al. Autosomal dominant cerebellar ataxia type I; Clinical features and MRI in families with SCA1, SCA2 and SCA3. Brain 1996; 119:1497-505.

5 Klockgether T, Skalej M, Wedekind D, et al. Autosomal dominant cerebellar ataxia type I; MRI-based volumetry of posterior fossa structures and basal ganglia in spinocerebellar ataxia types 1, 2 and 3. Brain 1998;121:1687-93.

6 Orr HT, Chung MY, Banfi S, et al. Expansion of an unstable trinucleotide CAG repeat in spinocerebellar ataxia type 1. Nat Genet 1993;4:221-6.

7 Kawaguchi $Y$, Okamoto T, Taniwaki $M$, et al. CAG expansions in a novel gene for Machado-Joseph disease at chromosome 14q32.1. Nat Genet 1994;8:221-8

8 Pulst SM, Nechiporuk A, Nechiporuk T, et al. Moderate expansion of a normally biallelic trinucleotide repeat in spinocerebellar ataxia type 2. Nat Genet 1996; 14:269-76.

9 Zhuchenko O, Bailey J, Bonnen $\mathrm{P}$, et al. Autosomal dominant cerebellar ataxia (SCA6) associated with small polyglutamine expansions in the $\alpha 1 \mathrm{~A}$-voltagedependent calcium channel. Nat Genet 1997;15:62-9.
10 Johansson J, Forsgren L, Sandgren O, et al. Expanded CAG repeats in Swedish spinocerebellar ataxia type 7 (SCA7) patients: effect of CAG repeat length on the clinical manifestation. Hum Mol Genet 1998;7:171-6.

11 Ito D, Yamada M, Kawai M, et al. Corneal endothelial degeneration in dentatorubral-pallidoluysian atrophy. Arch Neurol 2002;59:289-91.

12 Yoneda M, Tanno Y, Nonaka I, et al. Simple detection of tRNA(Lys) mutation in myoclonus epilepsy associated with ragged-red fibers (MERRF) by polymerase chain reaction with a mismatched primer. Neurology 1991;41:1838-40.

13 Nakamura $M$, Nakano $S$, Goto $Y$, et al. A novel point mutation in the mitochondrial tRNA ${ }^{\text {Ser(UCN) }}$ gene detected in a family with MERRF/MELAS overlap syndrome. Biochem Biophys Res Commun 1995;214:86-93.

14 Gilman S, Low PA, Quinn N, et al. Consensus statement on the diagnosis of multi system atrophy. J Neurol Sci 1999;163:94-8.

15 Whitwell JL, Crum WR, Watt HC, et al. Normalization of cerebral volumes by use of intracranial volume: implications for longitudinal quantitative MR imaging. ANNR Am J Neuroradiol 2001;22:1483-9.

16 David G, Durr A, Stevanin G, et al. Molecular and clinical correlation in autosomal dominant cerebellar ataxia with progressive macular dystrophy (SCA7). Hum Mol Genet 1998:7:165-70.

17 Ikeda K, Kubota S, Isashiki Y, et al. Machado-Joseph disease with retinal degeneration and dementia. Acta Neurol Scand 2001;104:402-5.

18 Sugawara M, Toyoshima I, Wada C, et al. Pontine atrophy in spinocerebellar ataxia type 6. Euro Neurol 2000;43:17-22.

19 Martin J, Van Regemorter N, Del-Favero J, et al. Spinocerebellar ataxia type 7 (SCA7) - correlation between phenotype and genotype in one large Belgian family. J Neurol Sci 1999;168:37-46

20 Martin JJ, Van Regemorter N, Krols L, et al. On an autosomal dominant form of retinal-cerebellar degeneration: an autopsy study of five patients in one family. Acta Neuropathol 1994;88:277-86.

21 Lindenberg KS, Yvert G, Muller K, et al. Expression analysis of ataxin-7 mRNA and protein in human brain: evidence for a widespread distribution and focal protein accumulation. Brain Pathol 2000;10:385-94.

22 Holmberg M, Duyckaerts C, Durr A, et al. Spinocerebellar ataxia type 7 (SCA7): a neurodegenerative disorder with neuronal intranuclear inclusions. Hum Mol Genet 1998:7:913-18.

23 Cancel G, Duyckaerts C, Holmberg M, et al. Distribution of ataxin-7 in normal human brain and retina. Brain 2000;123:2519-30.

24 De la Monte SM, Vonsattel JP, Richardson EP Jr. Morphometric demonstration of atrophic changes in the cerebral cortex, white matter, and neostriatum in Huntington's disease. J Neuropathol Exp Neurol 1988;47:516-25.

25 Ishikawa K, Watanabe M, Yoshizawa K, et al. Clinical, neuropathological, and molecular study in two families with spinocerebellar ataxia type 6 (SCA6) J Neurol Neurosurg Psychiatry 1999;67:86-9.

26 Sasaki H, Kojima H, Yabe I, et al. Neuropathological and molecular studies of spinocerebellar ataxia type 6 (SCA6). Acta Neuropathol 1998;95: 199-204

27 Sequeiros J, Coutinho P. Epidemiology and clinical aspects of MachadoJoseph disease. Adv Neurol 1993;61:139-53.

28 Coutinho P, Guimaraes A, Scaravilli F. The pathology of Machado-Joseph disease: report of a possible homozygous case. Acta Neuropathol (Berl) 1982;58:48-54.

29 Oh AK, Jacobson KM, Jen JC, et al. Slowing of voluntary and involuntary saccades: an early sign in spinocerebellar ataxia type 7. Ann Neurol 2001;49:801-4.

30 Lawrence $A D$, Hodges JR, Rosser $A E$, et al. Evidence for specific cognitive deficits in preclinical Huntington's disease. Brain 1998;121:1329-41.

31 Wadia N, Pang J, Desai J, et al. A clinicogenetic analysis of six Indian spinocerebellar ataxia (SCA2) pedigrees: the significance of slow saccades in diagnosis. Brain 1998;121:2341-55.

32 Gouw LG, Digre KB, Harris CP, et al. Autosomal dominant cerebellar ataxia with retinal degeneration: clinical, neuropathologic, and genetic analysis of a large kindred. Neurology 1994;44:1441-7.

33 Abe $Y$, Tanaka F, Matsumoto $M$, et al. CAG repeat number correlates with the rate of brainstem and cerebellar atrophy in Machado-Joseph disease. Neurology 1998;51:882-4. 